\title{
A CODING SCHEME FOR WIRELESS NETWORKS WITH MULTIPLE ANTENNA NODES AND NO CHANNEL INFORMATION
}

\author{
Frédérique Oggier and Babak Hassibi \\ Department of Electrical Engineering \\ California Institute of Technology \\ Pasadena, CA 91125 \\ \{frederique, hassibi\}@systems.caltech.edu
}

\begin{abstract}
In this paper, we present a coding strategy for wireless relay networks where the relay nodes are small devices with few resources, while the source and sink are equipped with multiple antennas to increase the transmission rate. We assume no channel knowledge at all, and the receiver decodes knowing none of the channel paths. This coding scheme uses distributed space-time coding techniques and is inspired by noncoherent differential space-time coding. It is shown to yield a diversity linear in the minimum number of transmit/receive antennas times the number of relays.
\end{abstract}

Index Terms - channel coding, mimo systems, networks.

\section{INTRODUCTION}

Coding for wireless networks has recently received a lot of attention. Using the knowledge acquired from space-time coding, where the transmit antennas cooperate to resist the fading, so-called cooperative diversity schemes techniques have been proposed, where the relay nodes form a virtual multiple antennas array to obtain the diversity advantage known to be achieved by MIMO systems [9, 1, 5, 13]. These works have focused on different aspects of wireless networks coding. In [5], distributed space-time codes (DSTC) are introduced, and their pairwise probability of error and the diversity gain computed. This work has been generalized in [6], for the case where the transmitter and receiver nodes both have several antennas. In [10], a construction of DSTCs for the multiple antenna case is presented.

All the above work assume some channel knowledge, either at the receiver, at the relays, or both. Coding strategies for the case when no channel information is available have been proposed independently in $[8,11,7]$. See also $[14,12]$ for different approaches to non-coherent DSTCs. All are based on noncoherent differential MIMO coding.

This work was supported by NSF grant CCR-0133818, by Caltech's Lee Center for Advanced Networking and by a grant from the David and Lucille Packard Foundation.
The goal of this work is to propose a noncoherent scheme for a wireless network where the transmitter and receiver nodes are equipped with multiple antennas. This generalizes the approach of [11] to the multiple antenna case. We start by recalling the multiple antenna nodes network model in Section 2. Section 3 presents the generalized differential scheme adapted for such networks. The diversity result is provided in Section 4.

\section{DISTRIBUTED SPACE-TIME CODING WITH MULTIPLE ANTENNAS}

Consider a wireless network as in [6], with $R+2$ randomly and independently distributed nodes. A source and a sink, equipped with several antennas, use the $R$ other nodes as relays. Every relay node has few resources and thus a single antenna. Denote the channels from the $M$ antennas of the transmitter to the $i$-th relay as $f_{1 i}, f_{2 i}, \ldots, f_{M i}$, and the channels from the $i$-th relay to the $N$ antennas at the receiver as $g_{i 1}, g_{i 2}, \ldots, g_{i N}$ (see Fig. 1). All channels are assumed iid $\mathcal{C N}(0,1)$. The total power of the system is denoted by $\tau$. It has been shown in [6] that the optimal power allocation is to have, for one transmission, $P_{1}=\tau / 2$ at the transmitter and $P_{2}=\tau /(2 R)$ at each relay. We also assume a coherence interval $T$ during which $f_{m i}$ and $g_{i n}$ are constant. The information bits are thus encoded into $T \otimes M$ matrices $\mathbf{S}=\left[\mathbf{s}_{1}, \ldots, \mathbf{s}_{M}\right]$, where $\mathbf{s}_{m}$ is a $T$-dimensional signal sent by the $m$-th transmit antenna. The signal $\mathbf{S}$ is normalized as

$$
\mathrm{E}\left[\operatorname{Tr}\left(\mathbf{S}^{\dagger} \mathbf{S}\right)\right]=M
$$

To send $\mathbf{S}$, we consider the two-step strategy of $[5,6]$.

Step 1: at the transmitter. From time 1 to $T$, the transmitter sends $\sqrt{P_{1} T / M} \mathbf{S}$. The received signal at the $i$-th relay at time $\tau$ is denoted by $r_{i \tau}$. Similarly, $v_{i \tau}$ denotes the additive noise at the $i$-th relay. The received signal at the relays is

$$
\mathbf{r}_{i}=\sqrt{P_{1} T / M} \sum_{m=1}^{M} f_{m i} \mathbf{s}_{m}+\mathbf{v}_{i}
$$




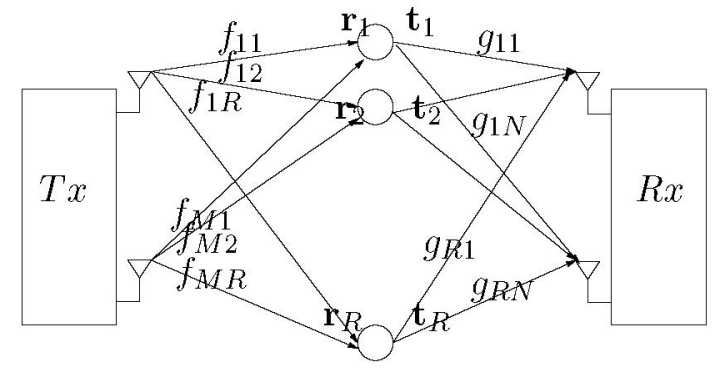

Fig. 1. The wireless network model with several antennas.

Step 2: at the relays. From time $T+1$ to $2 T$, the $i$-th relay sends $t_{i 1}, \ldots, t_{i T}$. The received signal and noise at the $n$-th antenna at time $T+\tau$ are denoted resp. by $x_{\tau n}$ and $w_{\tau n}$. The noises are iid $\mathcal{C N}(0,1)$. We have at the $n$th receive antenna

$$
\mathbf{y}_{n}=\sum_{i=1}^{R} g_{i n} \mathbf{t}_{i}+\mathbf{w}_{i} .
$$

The idea behind DSTBs is to design the transmit signal at every relay as a linear function of its received signal:

$$
\mathbf{t}_{i}=\sqrt{\frac{P_{2}}{P_{1}+1}} A_{i} \mathbf{r}_{i}, i=1, \ldots, R,
$$

where $A_{i}$ is a $T \otimes T$ unitary matrix (so that the protocol is equitable among different users and time instants, and the noise forwarded by the relays remains white). The normalization factor is such that the average transmit power is $P_{2} T$ at each relay (see [6]). If we summarize the channel model, denoting by $\mathrm{f}_{i}=\left[f_{1 i}, \ldots, f_{M i}\right]^{T}$, we get from (3) and (4) that

$\mathbf{y}_{n}=\sqrt{c_{\tau}}\left[A_{1} \mathbf{S} A_{R} \mathbf{S}\right]\left[\begin{array}{c}\mathbf{f}_{1} g_{1 n} \\ \vdots \\ \mathbf{f}_{R} g_{R n}\end{array}\right]+\sqrt{c_{\tau}^{\prime}} \sum_{i=1}^{R} g_{i n} A_{i} \mathbf{v}_{i}+\mathbf{w}$,

with $c_{\tau}=\frac{P_{1} P_{2} T}{\left(P_{1}+1\right) M}$ and $c_{\tau}^{\prime}=\frac{P_{2}}{P_{1}+1}$.

By defining $Y=\left[\mathbf{y}_{1}, \mathbf{y}_{2}, \ldots, \mathbf{y}_{N}\right], S=\left[A_{1} \mathbf{S} A_{2} \mathbf{S} \ldots A_{R} \mathbf{S}\right]$, $\mathbf{g}_{i}=\left[g_{i 1}, g_{i 2}, \ldots, g_{i N}\right], H=\left[\mathbf{f}_{1} \mathbf{g}_{1}, \ldots, \mathbf{f}_{R} \mathbf{g}_{R}\right]^{T}$, and

$W=\left[\sqrt{c_{\tau}^{\prime}} \sum_{i=1}^{R} g_{i 1} A_{i} \mathbf{v}_{i}+\mathbf{w}_{1}, \ldots, \sqrt{c_{\tau}^{\prime}} \sum_{i=1}^{R} g_{i N} A_{i} \mathbf{v}_{i}+\mathbf{w}_{N}\right]$, we finally get that the channel model can be written as

$$
Y=\sqrt{\frac{P_{1} P_{2} T}{\left(P_{1}+1\right) M}} S H+W
$$

where $Y$ is a $T \otimes N$ matrix, while $S$ is a $T \otimes M R$ matrix.

\section{A GENERALIZED DIFFERENTIAL SCHEME}

Let us now consider (5) and assume that the fadings are unknown. In a traditional noncoherent MIMO setting, it has been argued [2] that the codeword $S$ has to be unitary. To obtain a differential scheme, we follow the approach of [11]:

1. we design the matrices $A_{i}$ at the relays and an initial transmitted signal so that $S$ is unitary,

2. we define a differential encoder at the transmitter node.

\subsection{Designing the relays and an initial signal}

Recall that here

$$
S=\left[A_{1} \mathbf{S}, \ldots, A_{R} \mathbf{S}\right]
$$

The aim is to design the signal constellation $\left\{\mathbf{S}_{1}, \ldots, \mathbf{S}_{L}\right\} \ni$ $\mathbf{S}$ and the unitary matrices $A_{i}, i=1, \ldots, R$, so that the $T \otimes$ $M R$ matrix $S$ is unitary, i.e, $S^{\dagger} S=\mathbf{I}_{M R}$.

First, we choose the codewords to be of the form

$$
\mathbf{S}_{j}=U_{j} \mathbf{S}^{\prime}, j=1, \ldots, L
$$

where $U_{j}$ is a unitary $T \otimes T$ matrix, and $\mathbf{S}^{\prime}$ is an initial transmitted signal normalized so that $E\left[\operatorname{Tr}\left(\mathbf{S}_{j}^{\dagger} \mathbf{S}_{j}\right)\right]=M$ for all $j=1, \ldots, L$. Furthermore, we require that both the unitary matrices $A_{i}, i=1, \ldots, R$, and $U_{j}, j=1, \ldots, L$ are diagonal. Thus, denoting by $S_{j}=\left[A_{1} \mathbf{S}_{j}, \ldots, A_{R} \mathbf{S}_{j}\right]$, we have

$$
\begin{aligned}
S_{j}^{\dagger} S_{j} & =\left[A_{1} U_{j} \mathbf{S}^{\prime}, \ldots, A_{R} U_{j} \mathbf{S}^{\prime}\right]^{\dagger}\left[A_{1} U_{j} \mathbf{S}^{\prime}, \ldots, A_{R} U_{j} \mathbf{S}^{\prime}\right] \\
& =\left[A_{1} \mathbf{S}^{\prime}, \ldots, A_{R} \mathbf{S}^{\prime}\right]^{\dagger}\left[A_{1} \mathbf{S}^{\prime}, \ldots, A_{R} \mathbf{S}^{\prime}\right]
\end{aligned}
$$

and the fact that $S_{j}$ is unitary only depends on the choice of the matrices at the relays and the transmitted signal $\mathbf{S}^{\prime}$. We thus design the matrices $A_{i}$ and $\mathbf{S}^{\prime}$ independently of the codebook of matrices $U_{j}$.

Our construction is based on Butson-Hadamard matrices (eg. [3]). Recall that a Generalized Butson-Hadamard (GBH) matrix is a $T \otimes T$ matrix $\mathbf{M}$ with coefficients in a ring such that

$$
\mathbf{M M}^{*}=\mathbf{M}^{*} \mathbf{M}=T \mathbf{I}_{T}
$$

where $\mathbf{M}^{*}$ is the transpose of the matrix of inverse elements of $\mathbf{M}: m_{i j}^{*}=m_{j i}^{*}$. If the coefficients of $\mathbf{M}$ are chosen to be roots of unity, then $m_{i j}^{* 1}=\overline{m_{i j}}$, i.e., the inverse is the conjugate, so that

$$
\mathbf{M M}^{\dagger}=\mathbf{M}^{\dagger} \mathbf{M}=T \mathbf{I}_{T}
$$

Note that the tensor product of two GBH matrices is again a GBH matrix [3].

Suppose now that we have $T=M R$, with $M$ the number of transmit antennas, $R$ the number of relays, and $T$ the coherence time. Choose a first Butson-Hadamard matrix $A$ of size $R \otimes R$, and a second one $B$ of size $M$. Take

$$
A_{i}=\operatorname{diag}(\underbrace{a_{1 i}, \ldots, a_{1 i}}_{M}, \ldots, \underbrace{a_{R i}, \ldots, a_{R i}}_{M}) \text {, }
$$


which is unitary since all $a_{i j}$ 's are roots of unity, and similarly

$$
\mathbf{S}^{\prime}=\frac{\sqrt{M}}{\left\|\mathbf{S}^{\prime}\right\|_{F}}\left(\begin{array}{ccc}
b_{1,1} & \ldots & b_{1, M} \\
\vdots & & \vdots \\
b_{M, 1} & & b_{M, M} \\
\vdots & & \vdots \\
b_{1,1} & \ldots & b_{1, M} \\
\vdots & & \vdots \\
b_{M, 1} & & b_{M, M}
\end{array}\right),
$$

where $\frac{\sqrt{M}}{\left\|\mathbf{S}^{\prime}\right\|_{F}}$ is the normalization factor such that $E\left[\operatorname{Tr}\left(\mathbf{S}^{\prime \dagger} \mathbf{S}^{\prime}\right)\right]=$ $M$. By definition of the Frobenius norm,

$$
\left\|\mathbf{S}^{\prime}\right\|_{F}=\sqrt{\sum_{i=1}^{M} \sum_{j=1}^{T}\left|s_{i, j}^{\prime}\right|^{2}}=\sqrt{M T}
$$

since all coefficients $s_{i, j}^{\prime}$ are roots of unity. By construction

$$
\left[A_{1} \mathbf{S}^{\prime}, \ldots, A_{R} \mathbf{S}^{\prime}\right]=\frac{1}{\sqrt{T}} A \otimes B
$$

and

$$
\left[A_{1} \mathbf{S}^{\prime}, \ldots, A_{R} \mathbf{S}^{\prime}\right]^{\dagger}\left[A_{1} \mathbf{S}^{\prime}, \ldots, A_{R} \mathbf{S}^{\prime}\right]=\frac{R M}{T} \mathbf{I}_{R M}=\mathbf{I}_{R M},
$$

since $A \otimes B$ is a GBH matrix of size $R M$. For example, choose for $M=2$ and $R=3$ the following matrices:

$$
A=\left(\begin{array}{ccc}
1 & 1 & 1 \\
1 & \tau_{3} & \tau_{3}^{2} \\
1 & \tau_{3}^{2} & \tau_{3}
\end{array}\right) \text { and } B=\left(\begin{array}{cc}
1 & 1 \\
1 & \otimes 1
\end{array}\right)
$$

The unitary matrices at the relays are given by $A_{1}=\mathbf{I}_{6}$,

$A_{2}=\operatorname{diag}\left(1,1, \tau_{3}, \tau_{3}, \tau_{3}^{2}, \tau_{3}^{2}\right), A_{3}=\operatorname{diag}\left(1,1, \tau_{3}^{2}, \tau_{3}^{2}, \tau_{3}, \tau_{3}\right)$,

and the initial transmitted signal is

$$
\mathbf{S}^{\prime}=\sqrt{\frac{2}{12}}\left(\begin{array}{cccccc}
1 & 1 & 1 & 1 & 1 & 1 \\
1 & \otimes 1 & 1 & \otimes 1 & 1 & \otimes 1
\end{array}\right)^{T} .
$$

\subsection{A Differential Encoder}

The two-step transmission of Section 2 can be adapted to allow for differential encoding and decoding as follows. Let $U\left(z_{t+n T}\right)$ be the unitary matrix that encodes the data $z_{t+n T}$ (to be sent at time $t+n T$ ).

Step 1: at the transmitter. The transmitter sends consecutively $\mathbf{S}_{t}=U\left(z_{t}\right) \mathbf{S}^{\prime}$ and $\mathbf{S}_{t+T}=U\left(z_{t+T}\right) \mathbf{S}_{t}$. At the $i$ th relay, the received signals are

$$
\begin{aligned}
\mathbf{r}_{i}(t) & =\sqrt{P_{1} T / M} \mathbf{S}_{t} \mathbf{f}_{i}+\mathbf{v}_{i}(t) \\
\mathbf{r}_{i}(t+T) & =\sqrt{P_{1} T / M} U\left(z_{t+T}\right) \mathbf{S}_{t} \mathbf{f}_{i}+\mathbf{v}_{i}(t+T)
\end{aligned}
$$

where $\mathbf{f}_{i}=\left[f_{1 i}, \ldots, f_{M i}\right]^{T}, i=1, \ldots, R$.

Step 2: at the relays. Each relay $R_{i}, i=1, \ldots, R$, consecutively multiplies its received signal by a unitary matrix $A_{i}\left(A_{i}\right.$ is built as described above) and then transmit $\mathbf{t}_{i}(t)=$ $\sqrt{\frac{P_{2}}{P_{1}+1}} A_{i} \mathbf{r}_{i}(t)$, and $\mathbf{t}_{i}(t+T)=\sqrt{\frac{P_{2}}{P_{1}+1}} A_{i} \mathbf{r}_{i}(t+T)$. The received signals are similar to $(5)$ :

$$
\begin{aligned}
Y(t) & =\sqrt{c_{\tau}}\left[A_{1} \mathbf{S}_{t} A_{2} \mathbf{S}_{t} \ldots A_{R} \mathbf{S}_{t}\right] H+W(t) \\
Y(t+T) & =\sqrt{c_{\tau}} U\left(z_{t+T}\right)\left[A_{1} \mathbf{S}_{t} \ldots A_{R} \mathbf{S}_{t}\right] H+W(t+T),
\end{aligned}
$$

where last equation holds since $A_{i}$ and $U\left(z_{t+T}\right)$ commute, for all $i$ and for all $U\left(z_{t+T}\right)$. We can thus plug the equation for $Y(t)$ into the one of $Y(t+T)$, which yields

$$
Y(t+T)=U\left(z_{t+T}\right) Y(t)+W(t, t+T),
$$

where

$$
W(t, t+T)=\otimes U\left(z_{t+T}\right) W(t)+W(t+T)
$$

is the noise term, and $W(t), W(t+T)$ are given as in (5). Note that the channel coefficients $\mathbf{f}_{i}, \mathbf{g}_{i}$ do not appear in (6), meaning that one can decode without knowing the channel.

\section{DIVERSITY COMPUTATION}

Emulating the point to point case, a natural candidate for the differential decoder is

$$
\arg \min _{U_{l}, l=1, \ldots, L}\left\|Y(t+T) \otimes U_{l} Y(t)\right\|^{2} .
$$

Let us restrict to the case where $T=M R$. We consider two instances of the noncoherent channel (5)

$$
Y(t)=\sqrt{c_{\tau}} S(t) H+W(t)
$$

where $H$ is an $T \otimes N$ matrix unknown at both the transmitter and receiver. Since $S(t)$ and $S(t) \tau$ are indistinguishable for an arbitrary unitary $T \otimes T$ matrix $\tau$, we preprocess the signal so that

$$
\left(\begin{array}{c}
Y(t) \\
Y(t+T)
\end{array}\right)=\sqrt{c_{\tau}}\left(\begin{array}{c}
\mathbf{I}_{T} \\
U_{k}
\end{array}\right) H+\left(\begin{array}{c}
W(t) \\
W(t+T)
\end{array}\right),
$$

for $U_{k}$ a unitary matrix of the codebook. Because of the two steps transmission, both the noise $W(t)$ and the channel matrix $H$ contains products of Gaussian random variables, which makes a precise analysis difficult. Following [11], we consider a mismatched decoder, and claim that such a decoder actually already gives the diversity. More precisely, we can prove that the diversity gain of the coding strategy described above is

$$
d= \begin{cases}\min \{M, N\} R & M \neq N \\ M R\left(1 \otimes \frac{1}{M} \frac{\log \log P}{\log P}\right) & M=N,\end{cases}
$$




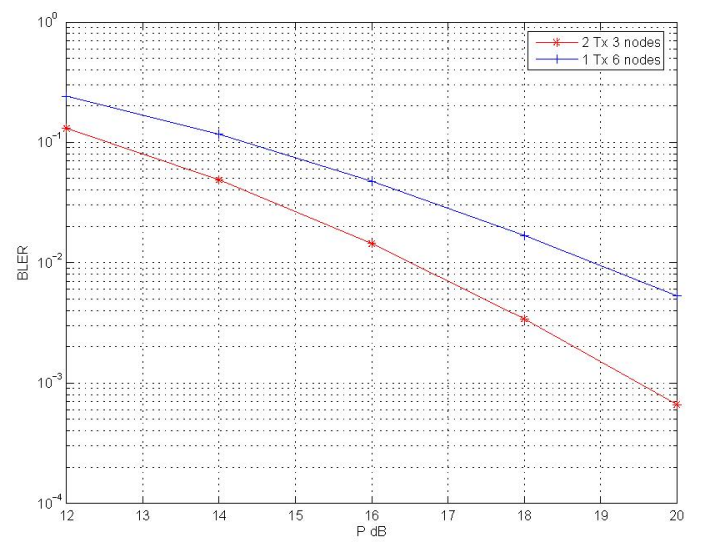

Fig. 2. A 6 nodes network with 1 antenna versus a 3 nodes network with 2 antennas.

assuming that $\left(U_{k} \otimes U_{l}\right)\left(U_{k} \otimes U_{l}\right)^{\dagger}$ is full rank (that is, the code is fully diverse). The proof is omitted for lack of space, but we briefly outline the main steps. We are interested in computing the pairwise probability of the above channel, i.e.,

$$
P\left(U_{k} \rightarrow U_{l}\right)=E_{\mathbf{g}}\left[P\left(U_{k} \rightarrow U_{l} \mid \mathbf{g}\right)\right], k \neq l .
$$

The main difficulty compared to the work in [11] is that the columns of the channel matrix $H$ are not independent anymore, and the matrix $H$ is not full rank. Thus the way of doing the computation is to actually vectorize the system, and then compute the pairwise probability of error knowing $\mathrm{g}$. This computation relies on the technique that consists of computing the probability in the complex domain to avoid convergence issue, and then, knowing that the probability is real, in using the real part of the expression to get an upper bound. Once this bound on the probability is computed, we rely on the computation of the expectation on $\mathrm{g}$ done in [6, Theorem 3], from which follows the diversity gain.

What the diversity computation tells us is that the diversity is linear in the number of relays times the minimum number of transmit/receive antennas. This is illustrated in Fig. 2, where the BLER of a 6 nodes network with one antenna at transmitter and receiver is compared to a 3 nodes network with 2 antennas at both transmitter and receiver nodes. Note that both networks use the same codebook, meaning that the 2 antennas network is operating at half the rate. The codebook used here is a cyclic code [2]. As shown above, the design of the codebook is an independent issue.

\section{CONCLUSION}

We considered the problem of coding over a wireless relay network, where the transmitter and receiver nodes are equipped with several antennas to increase the rate of the network. We furthermore assumed that there is no channel information, and that the receiver has to decode without knowing the channel paths during communication. In order to propose a coding strategy, we generalized the concept of differential distributed space-time coding, and showed that we expect a diversity that is linear in the minimum number of antennas at the transmitter/receiver, times the number of relays.

\section{REFERENCES}

[1] K. Azarian, H. El Gamal, P. Schniter, "On the achievable diversity-multiplexing tradeoff in half-duplex cooperative channels,"IEEE Trans. Inf. Theory, vol. 51, Dec. 05.

[2] B. Hochwald,W. Sweldens, "Differential unitary space time modulation", IEEE Trans.Comm,vol.48,Dec.2000.

[3] K. J. Horadam, "A generalised Hadamard transform", in the proceedings of ISIT 2005, Adelaide.

[4] B. Hughes, "Differential space-time modulation," IEEE Trans. Inform. Theory, vol 46, Nov. 2000.

[5] Y. Jing and B. Hassibi, "Distributed space-time coding in wireless relay networks," IEEE Trans. on Wireless Communications, vol. 5, no 12, Dec. 2006.

[6] Y. Jing and B. Hassibi, "Cooperative Diversity in Wireless Relay Networks with Multiple-Antenna Nodes", submitted to IEEE Transactions on Signal Processing.

[7] Y. Jing and H. Jafarkhani, “ Distributed Differential Space-Time Coding for Wireless Relay Networks", submitted to IEEE Trans. on Communications.

[8] T. Kiran and B. Sundar Rajan, " Partially-coherent distributed space-time codes with differential encoder and decoder," in the proceedings of ISIT 2006, Seattle.

[9] J. N. Laneman and G. W. Wornell, "Distributed SpaceTime Coded Protocols for Exploiting Cooperative Diversity in Wireless Networks," IEEE Trans. Inf. Theory, vol. 49, no 10, Oct. 2003.

[10] F. Oggier, B. Hassibi,"An Algebraic Coding Scheme for Wireless Relay Networks with Multiple-Antenna Nodes", subm. to IEEE Trans. Signal Proc., Mar. 2006.

[11] F. Oggier, B. Hassibi, "A Coding Strategy for Wireless Networks with no Channel Information", Allerton06.

[12] T. Wang, Y. Yao, G. B. Giannakis, "Non-Coherent Distributed Space-Time Processing for Multiuser Cooperative Transmissions," Proc. of Globecom Conf. 2005.

[13] S. Yang and J.-C. Belfiore, "Optimal Space-Time Codes for the Amplify-and-Forward Cooperative Channel, " IEEE Trans. Inform. Theory,vol. 53, no 2, Feb 2007.

[14] S. Yiu, R. Schober, L. Lampe, "Differential Distributed Space-Time Block Coding”, IEEE PacRim conf., 2005. 